\title{
Herpesvirus saimiri-mediated delivery of the adenomatous polyposis coli tumour suppressor gene reduces proliferation of colorectal cancer cells
}

\author{
STUART A. MACNAB ${ }^{1,2}$, SUSAN J. TURRELL ${ }^{1,2}$, IAN M. CARR ${ }^{3}$, \\ ALEX F. MARKHAM $^{3}$, P. LOUISE COLETTA ${ }^{3}$ and ADRIAN WHITEHOUSE ${ }^{1,2}$ \\ ${ }^{1}$ Institute of Molecular and Cellular Biology, Faculty of Biological Sciences, and ${ }^{2}$ Astbury \\ Centre for Structural Molecular Biology, University of Leeds, Leeds, LS2 9JT; ${ }^{3}$ Leeds Institute \\ of Molecular Medicine, St. James University Hospital, Leeds, LS9 7TF, UK
}

Received April 29, 2011; Accepted June 2, 2011

DOI: 10.3892/ijo.2011.1130

\begin{abstract}
Colorectal cancer (CRC) is a major cause of cancerrelated mortality. A contributing factor to the progression of this disease is sporadic or hereditary mutation of the adenomatous polyposis coli (APC) gene, a negative regulator of the Wnt signalling pathway. Inherited mutations in APC cause the disorder familial adenomatous polyposis (FAP), which leads to CRC development in early adulthood. However, the gene is also disrupted in some $60 \%$ of sporadic cancers. Restoration of functional APC may slow the growth of CRC by negatively regulating proliferation-associated genes such as c-myc. Therefore, we have cloned the cDNA of the APC tumour suppressor gene into a replication competent Herpesvirus saimiri (HVS)-based vector to assess APC gene delivery in SW480 and SW620 CRC cell lines. Our results demonstrate that full length APC protein was efficiently expressed from the HVS vector and that transgene expression inhibited proliferation of both the SW480 and the metastatic SW620 cancer cell lines. Moreover, a sustained effect could be observed for at least 8 weeks after initial infection in SW480 cells. In addition, monolayer wounding assays showed a marked reduction in proliferation and migration in HVS-GFP-APC infected cells. We believe that this is the first instance of infectious delivery and APC cDNA expression from a virus-based vector.
\end{abstract}

\section{Introduction}

In 2008 colorectal cancer (CRC) was the third leading cause of cancer-related deaths in both women and men, with a respective incidence of $10 \%$ and $11 \%$ of all cancers in the UK (http:// info.cancerresearchuk.org/cancerstats). There are several risk

Correspondence to: Dr Adrian Whitehouse, Institute of Molecular and Cellular Biology, University of Leeds, LS2 9JT, Leeds, UK

E-mail: a.whitehouse@leeds.ac.uk

Key words: adenomatous polyposis coli, gene therapy factors associated with CRC, including age, obesity, smoking, diet and inflammatory bowel disease (1). Other high risk groups are those suffering from inherited diseases, such as Lynch syndrome and familial adenomatous polyposis (FAP) (2).

FAP is an autosomal dominantly inherited syndrome associated with an increased susceptibility to colorectal neoplasia, with $100 \%$ of sufferers going on to develop cancer if untreated. This disease is characterised by the presence of hundreds to thousands of pre-malignant adenomatous polyps in the colon. Polyps develop from early adolescence and progress to CRC in early adulthood (3). The molecular mechanism behind this syndrome is mutation of the tumour suppressor gene adenomatous polyposis coli (APC), located on chromosome $5 \mathrm{q} 21(4,5)$.

APC is a $312-k D a$ multi-domain homodimeric protein with a myriad of functions, including cell cycle regulation and control of chromosome segregation and cell adhesion and migration (6,7). Moreover, it carries out an important function in epithelial cell biology; to act as a component of the Wnt signalling pathway $(8,9)$. APC forms a cytoplasmic complex with the scaffold protein axin and the kinase GSK $3 \beta$. This destruction complex binds and phosphorylates $\beta$-catenin, leading to its ubiquitination and subsequent degradation (9). However when the Wnt pathway is activated, the protein Dishevelled inhibits the formation of this complex, in turn preventing phosphorylation and degradation of $\beta$-catenin. This allows transient activation of $\beta$-catenin-responsive genes. Mutation of APC leads to constitutive activation of the Wnt pathway as $\beta$-catenin is not efficiently degraded, and continuously up-regulates gene expression by interaction with the T-cell family of transcription factors (TCF). $\beta$-catenin-responsive genes affect cellular proliferation, cell survival and apoptosis avoidance and include c-myc, cyclin D1 and components of the AP-1 transcription factor complex (10-12).

APC is a large gene, encoding an 8.5-kb cDNA from a $98-\mathrm{Kb}$ genomic locus, spanning 15 exons. A region of exon 15 , termed the mutation cluster region, is the site of the majority of APC mutations, frequently resulting in truncated versions of the protein $(13,14)$. APC mutations are inherited in FAP patients but have also been detected in some $60 \%$ of sporadic colorectal cancers, and have been identified as one of several 
series of key mutations in the well-defined progression of this disease $(13,15)$.

As APC mutation is such a crucial factor in CRC progression, restoration of the functional protein is an attractive concept to halt advancement of this disease. To date, delivery of an APC transgene has only been achieved in vivo by using non-viral gene therapy vectors. Westbrook et al demonstrated that by enema delivery, human exogenous APC could produce prolonged gene expression in rodent rectal epithelium (16), and Hargest et al further advanced this work by showing APC gene expression at higher sights in colonic crypts than the natural enterocyte turnover point, suggesting that crypt stem cells could be transfected (17). A recent study using a liposome vector showed that APC gene replacement therapy reduced small bowel polyposis in a mouse model by $25 \%$ (18), demonstrating a therapeutic in vivo application of APC gene therapy. Following this, a myc-tagged APC construct and liposome-mediated delivery system was used to stably express APC cDNA and led to reduction in the proliferation of transfected cells (19). These achievements demonstrate the clinical potential of restoring APC expression, but liposome-mediated delivery is an inefficient administration method and efficacy could be improved with the use of safe viral gene therapy vectors.

Herpesvirus saimiri (HVS) is a novel gene therapy vector with several favourable traits $(20,21)$. This $\gamma$-2 herpes virus has a large transgene capacity, making it a practical tool for delivering large gene sequences. It exists as a stable non-integrated episome in the host nucleus and therefore has a very low risk of insertional mutagenesis. In addition, HVS can efficiently infect a range of human cells including those from erythroid, epithelial and connective tissues lineages. Infection has been demonstrated in cell lines derived from colorectal cancers, in both in vitro and in vivo studies, and the virus is maintained in dividing cell populations, prolonging transgene expression (22-28).

One such cell line, SW480, has a mutant version of the APC tumour suppressor gene, causing a premature termination of the protein at amino acid 133. It is therefore a model cell line for studying APC function $(29,30)$. HVS has shown high tropism for SW480 cells in vitro and can efficiently infect SW480-derived xenograph and multicellular spheroids Moreover, it exists in a non-integrated, episomally maintained, latent state (28), suggesting that the virus would make a suitable vector to mediate APC gene therapy.

Herein, we have produced the first HVS-based viral vector expressing an APC transgene. HVS-GFP-APC expresses APC cDNA under the control of a thymidine kinase promoter fused to multiple transcription factor (TCF) 4 response elements (termed the APC mini-gene). This expression cassette is designed to provide native levels of APC, due to a negative feedback loop. The HVS-GFP-APC vector restored functional APC expression in SW480 cells, leading to reduced growth rates in vitro. Moreover, APC was stably expressed for at least an 8-week period in these cells and also slowed proliferation in the more aggressive SW620 cell line, illustrating the therapeutic potential of this novel gene therapy system.

\section{Materials and methods}

Cell culture and transfection. The owl monkey kidney (OMK) packaging cell line, and colorectal carcinoma cell lines SW480 and HCT116 were maintained in Dulbecco's modified Eagle's medium (DMEM) (Invitrogen), supplemented with $10 \%$ foetal calf serum (FCS) (Gibco) and $5 \mathrm{U} / \mathrm{ml}$ penicillin and streptomycin (Gibco). Colorectal carcinoma cell line SW620 was grown in L-15 medium (Sigma) supplemented with 10\% FCS. All cells were maintained in a humidified atmosphere containing $5 \%$ $\mathrm{CO}_{2}$. Transfections were carried out in 6-well 35-mm dishes with Lipofectamine 2000 (Invitrogen), when cells were $75 \%$ confluent. Typically $3 \mu \mathrm{g}$ DNA and $3 \mu$ l Lipofectamine 2000, respectively, were added into separate tubes of $250 \mu \mathrm{l} \mathrm{FCS-free}$ DMEM and incubated for $5 \mathrm{~min}$. DNA and Lipofectamine solutions were then mixed and incubated for 20 min to allow formation of DNA/lipid complexes. The DNA/lipid solution was then added to the cells, which were incubated for $16 \mathrm{~h}$ at $37^{\circ} \mathrm{C}$ in DMEM supplemented with $2.5 \%$ (v/v) FCS as well as $5 \mathrm{U} / \mathrm{ml}$ penicillin and streptomycin. Post incubation, the media were replaced with DMEM containing $10 \%$ (v/v) FCS.

Construction of APC-expressing HVS vectors. The HVSGFP-BAC utilised is a recombinant strain of HVS A11-S4 with a bacterial artificial chromosome (BAC) cassette inserted into ORF 15 (31,32). The BAC cassette confers chloramphenicol and hygromycin resistance, and contains a GFP expression marker and a unique I-PpoI restriction site. This virus has been shown to have an identical in vitro phenotype to the wild-type strain of HVS A11-S4. pShuttle-Link1-APC contains the 8.5-kb APC cDNA, under the control of a thymidine kinase promoter fused to multiple transcription factor (TCF) 4 response elements. The parent vector from which pShuttle-Link1-APC was derived (pShuttle-Link1) was described previously (31).

The recombinant viruses, HVS-GFP-APC-4 and HVSGFP-APC-5, were constructed by insertion of the APC expression cassette from pShuttle-Link1-APC into HVSGFP-BAC. This was achieved by a simple cloning procedure, briefly, HVS-GFP-BAC was linearised by I-PpoI digestion, the enzyme was then heat inactivated and salts were removed from the sample by drop dialysis using a $0.025-\mu \mathrm{m}$ nitrocellulose membrane filter (Millipore). The APC expression cassette was excised from pShuttle-Link1-APC by I-PpoI digestion and purified by gel extraction (Invitrogen). Ligation reactions were carried out overnight in an ice bath with the temperature increasing to room temperature. After a further drop dialysis, ligations were transformed into DH10 $\beta$ electrocompetent $E$. coli according to the manufacturer's instructions (Invitrogen) and plated onto LB agar containing $12 \mu \mathrm{g} / \mathrm{ml}$ chloramphenicol and $25 \mu \mathrm{g} / \mathrm{ml}$ kanamycin. Positive clones were analysed by pulse field gel electrophoresis of purified and digested viral DNA.

Virus production. Infectious viral particles were obtained by transfection of HVS DNA into permissive OMK cells. After transfection, cells were grown in DMEM supplemented with $5 \%(\mathrm{v} / \mathrm{v}) \mathrm{FCS}$ as well as $5 \mathrm{U} / \mathrm{ml}$ penicillin and streptomycin to enhance viral infection of neighbouring cells. Once the cell sheet was fully lysed, the supernatant from the 6-well plate was used to infect and lyse OMK cells in a $75-\mathrm{cm}^{2}$ tissue culture flask to amplify virus stocks.

Virus infection and stable cell line formation. Infections were performed in 6-well plates with cells at $70 \%$ confluency. Virus 
at a m.o.i of 1-5 was added directly to the culture medium (DMEM supplemented with $5 \%(\mathrm{v} / \mathrm{v})$ FCS as well as $5 \mathrm{U} / \mathrm{ml}$ penicillin and streptomycin), $24 \mathrm{~h}$ after cell seeding. Infection could be visualised via GFP reporter gene expression. Stable cell lines were formed by the addition of $4 \mu \mathrm{l} / \mathrm{ml}$ hygromycin B (Sigma) $48 \mathrm{~h}$ post infection, selecting for infected cells.

Episome rescue. BAC DNA was isolated using the HIRT extraction procedure. Approximately $1 \times 10^{6}$ cells containing the HVS episome were trypsinised and washed twice in $2 \mathrm{ml}$ PBS (Invitrogen). Cell pellets were then re-suspended in $50 \mu \mathrm{l}$ STET buffer [0.1 M NaCl, $10 \mathrm{mM}$ Tris (pH 8.0), 1 mM EDTA, $5 \%(\mathrm{v} / \mathrm{v})$ Triton X-100] and incubated at room temperature for 5 min. Cell lysis was performed via incubation of the solution on ice with $130 \mu 110 \%$ (w/v) SDS solution for $5 \mathrm{~min}$. Samples were neutralised with $110 \mu \mathrm{l} 5 \mathrm{M}$ ammonium acetate and incubated on ice for a further $5 \mathrm{~min}$. Cell debris was removed via centrifugation at 7,000 for $10 \mathrm{~min}$. The supernatant was harvested using a cut pipette tip to avoid sheering of the BAC episome. DNA was purified using $400 \mu \mathrm{l}$ phenol:chloroform:isoamyl alcohol (25:24:1) (Sigma) and centrifugation at 9,000 rpm for $15 \mathrm{~min}$. The BAC DNA was precipitated from the solution with $1 \mathrm{ml}$ ice-cold $100 \%$ ethanol and a 30-min incubation at $-20^{\circ} \mathrm{C}$, then pelleted via centrifugation at $10,000 \mathrm{rpm}$. Excess salts were removed from the DNA pellet via a 1-ml wash with ice-cold $70 \%(\mathrm{v} / \mathrm{v})$ ethanol and centrifugation at 10,000 rpm for $10 \mathrm{~min}$. DNA pellets were gently re-suspended using a cut

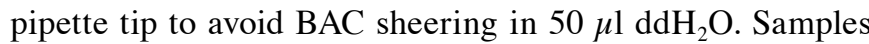
were then drop dialysed and stored at $4^{\circ} \mathrm{C}$.

Pulsed field gel electrophoresis. A Bio-Rad pulsed field gel electrophoresis (PFGE) tank was filled with 2.5 1, 0.5X TBE buffer (0.0225 M Tris-borate, 0.001 M EDTA). The buffer was pre-chilled using a Bio-Rad Model 1000 mini chiller programmed at $15.5^{\circ} \mathrm{C}$ and circulated with a Bio-Rad variable speed pump. The 1.2\% PFGE grade agarose gel was loaded with the ladders Lambda HindIII (Promega) and MidRange I PFG or II PFG Marker (New England Biolabs), and the BAC DNA samples. The Bio-Rad Chef DR II drive module was programmed with the following conditions; 6 volts, $16.2 \mathrm{~h}$, initial SW 2.0, final SW 16.0. After allowing the samples to run into the gel for $\sim 20 \mathrm{~min}$, the cooling unit and variable speed pump were switched back on. Gels were stained subsequent to electrophoresis using $200 \mathrm{ml} 0.5 \mathrm{X}$ TBE with $0.001 \mathrm{mg} / \mathrm{ml}$ ethidium bromide (Sigma).

Immunofluorescence. Approximately $5 \times 10^{5}$ SW480 or HCT116 cells were seeded onto ethanol-cleaned coverslips in $35-\mathrm{mm}$ 6-well tissue culture plates. After incubation for 6-12 h cells were infected separately overnight with HVS-GFP-BAC, HVS-GFP-APC4 or HVS-GFP-APC5 at a m.o.i of 0.25 . Culture medium was then replaced with DMEM supplemented with $10 \%$ (v/v) FCS as well as $5 \mathrm{U} / \mathrm{ml}$ penicillin and streptomycin, for a further $24 \mathrm{~h}$. Prior to fixation, growth media were removed and cells gently washed with PBS (Invitrogen). After fixation in $4 \%(\mathrm{v} / \mathrm{v})$ formaldehyde in PBS solution for $10 \mathrm{~min}$ at $20^{\circ} \mathrm{C}$, cells were washed $3 \mathrm{X}$ with PBS and permealised with $0.5 \%(\mathrm{v} / \mathrm{v})$ Triton X-100 in PBS for $10 \mathrm{~min}$. Cells were then washed a further $3 \mathrm{X}$ with PBS. Immunofluorescence staining was carried out on fixed cells immediately. Blocking took place for $1 \mathrm{~h}$ at $37^{\circ} \mathrm{C}$ using a $1 \%(\mathrm{v} / \mathrm{v})$ bovine serum albium (BSA) in PBS. Blocking solution was then removed and cells were incubated with primary rat C-terminal antibodies (KT45, Absea) diluted 1:250 in blocking solution. Samples were incubated at $37^{\circ} \mathrm{C}$ for $1 \mathrm{~h}$. Primary antibody solution was then removed and cells were washed five times in PBS. Cells were then incubated with the secondary anti-rat antibody conjugated to Alexa Fluor 546 (Invitrogen) diluted at a concentration of 1:500 in $1 \%(\mathrm{v} / \mathrm{v}) \mathrm{BSA}$ PBS solution at $37^{\circ} \mathrm{C}$ for $1 \mathrm{~h}$. Cover slips were washed five times using PBS. Samples were mounted onto glass slides using VectorShield with DAPI (Laboratories Inc.) and stored in a light resistant container at $4^{\circ} \mathrm{C}$ until required. Immunofluorescence was visualised on an inverted Zeiss LSM 520 Meta confocal microscope system and using LSM 510 Meta software.

Cellular proliferation assays. SW480 cells were seeded into 6 -well plates at $1 \times 10^{4}$ cells per well. Cells were incubated in $10 \%$ DMEM and cell counts were carried out at $24 \mathrm{~h}$ intervals. Prior to cell counts, cells were detached from the tissue culture dish with $0.25 \%$ trypsin (Invitrogen). Cells were then diluted in Trypan-blue solution (Sigma) at dilution factors ranging from 2-8, before being counted on a Neubauer Improved $0.1 \mathrm{~mm}$ depth haemocyto-meter with $22 \times 25 \times 0.5 \mathrm{~mm}$ coverslips (Hawksley).

Scratch assays. Assays were performed in 6-well plates where SW480 cells had reached $100 \%$ confluence. Monolayer wounding was carried out using a 1-ml Gilson pipette tip (Starlabs), by scratching along the diameter of the dish whilst in $10 \%$ DMEM. Media were removed and the remaining cells were washed with PBS (Invitrogen) until all cellular debris had been removed from the tissue culture dish. Cells were then incubated in 10\% DMEM throughout the course of the experiment. Images of cellular re-growth into the wound were carried out at $24 \mathrm{~h}$ intervals. Images were recorded using a Leica DC 300F camera with IM50 software.

\section{Results}

Construction of HVS-GFP-APC vectors. The HVS-GFP-BAC allows heterologous gene expression cassettes to be easily inserted into HVS-based vectors in a non-disrupting, sitespecific manner. pShuttle-Link1-APC contained the APC mini-gene and a kanamycin selection marker flanked by I-PpoI restriction sites. The 10-kb APC mini-gene from pShuttleLink1-APC was cloned into the HVS-GFP-BAC via its unique I-PpoI site (Fig. 1A). Positive colonies from the transformed ligations were screened via restriction digestion with I-PpoI, which excises the 10-kb mini-gene from the linearised 174-kb BAC plasmid. DNA was visualised by pulse field gel electrophoresis (PFGE) (Fig. 1B). PFGE indicated that a number of recombinant HVS-GFP-BAC genomes contained the APC mini-gene DNA. These genomes were designated via colony number and the prefix; HVS-GFP-APC. The resulting genome has an approximate size of $184 \mathrm{~kb}$. Clones 4 and 5 were selected for further analysis.

HVS-GFP-APC clones 4 and 5 were transfected into OMK cells to assess whether the increase in genome size-affected virus production. GFP fluorescence was utilised to monitor 
A

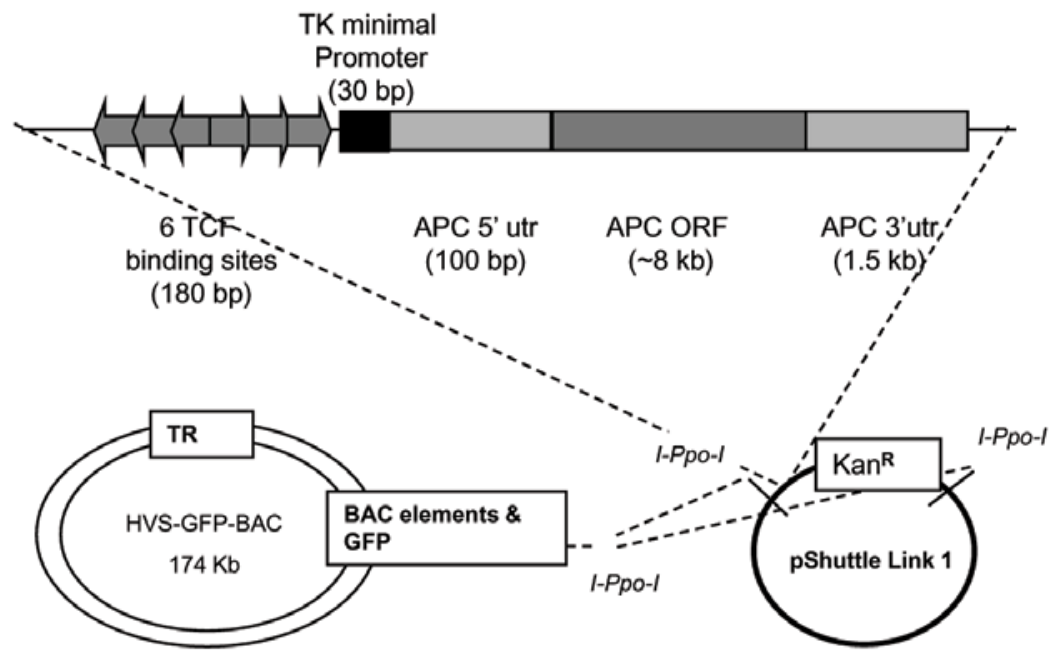

B

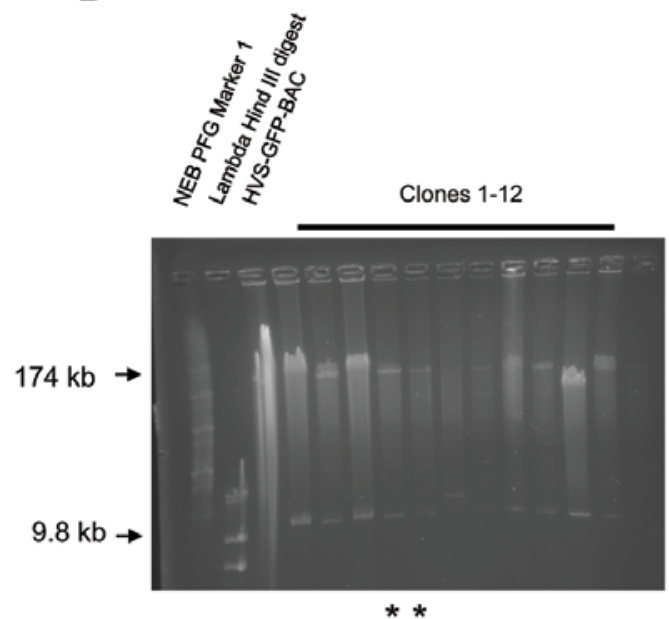

C

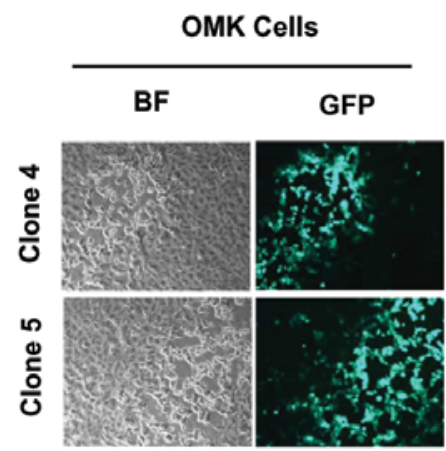

Figure 1. Construction of HVS-GFP-APC vectors. (A) The APC mini-gene comprises the APC cDNA under the control of a TCF-regulated thymidine kinase (TK) minimal promoter. As the APC-Axin-GSK3 $\beta$ complex is involved in the degradation of $\beta$-catenin (a protein involved in TCF-responsive promoter activity), this acts as a natural negative regulatory mechanism. The unique I-Ppo-I restriction sites contained within the HVS-BAC genome and pShuttleLink1-APC allow site-specific insertion of the APC mini-gene into the HVS genome. (B) Restriction analysis of potential HVS-GFP-APC clones using I-PpoI reveals that all tested clones contain the HVS-GFP-BAC backbone and APC mini-gene insert. "Clones 4 and 5 were selected for further analysis. (C) The HVS-permissive cell line, OMK, was transfected with HVS-GFP-APC DNA to produce infectious viral particles (clones 4 and 5). BF, bright field; GFP, cells stimulated via UV to emit green fluorescence. Images taken 4 days post-transfection.

virus production. After $48 \mathrm{~h}$ a number of OMK cells expressed GFP and after 7 days GFP-positive viral plaques were observed (Fig. 1C) which eventually ablated the cell sheet. The successful production of the HVS-GFP-APC viruses indicated that this vector could be utilised to deliver the APC mini-gene. The medium harvested from the lysed cells was used to infect naïve OMK cells, to further amplify virus stocks.

APC protein is expressed from HVS-GFP-APC vectors. To determine if HVS-based delivery of APC cDNA resulted in full-length APC expression, the protein was visualised by immunofluorescence (IF) in the model SW480 cell line, prior to assessing any therapeutic effects. HVS-GFP-BAC virus or HVS-GFP-APC (clones 4 and 5) was used to infect SW480 cells at a m.o.i of $\sim 0.25$. HCT116 colorectal cancer cells were used as a positive control for APC protein visualisation. An APC-specific antibody raised against the carboxyl terminus of APC was used, as SW480 cells contain an N-terminal-truncated version of the APC protein. Therefore, any positive IF in these cells is due to expression from the HVS-delivered APC mini-gene.

The IF of endogenous APC expression in both cell lines demonstrated that full-length APC is present in HCT116 cells (Fig. 2K), whereas no full-length APC was detected in the SW480 cells (Fig. 2L). Similarly, when SW480 cells were infected with HVS-GFP-BAC virus, the C-terminal antibody did not detect APC (Fig. 2M). In contrast, APC expression was observed in HVS-GFP-APC infected cells (Fig. 2N and O). This demonstrates that the complete APC mini-gene can be 

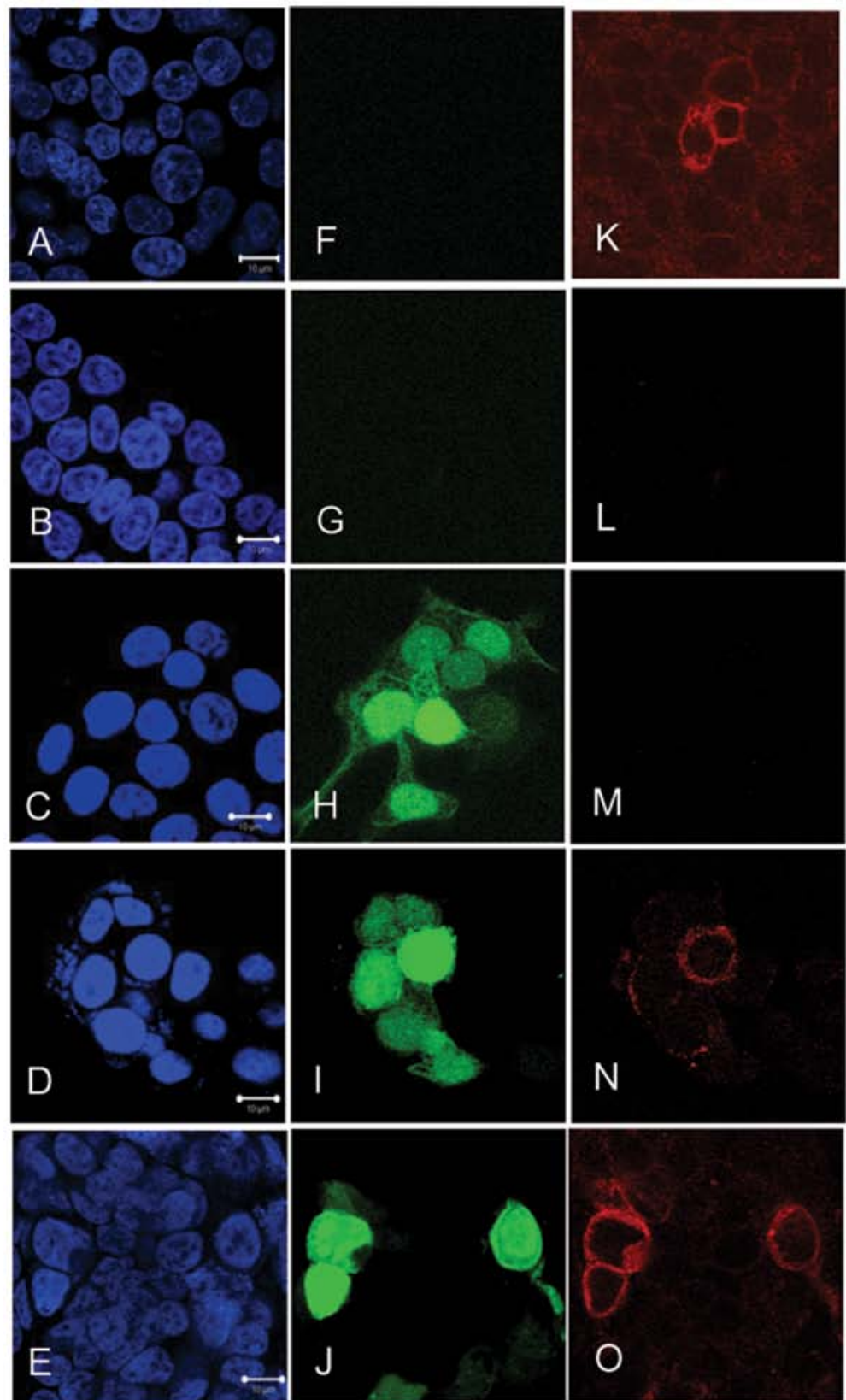

Figure 2. Immuno-fluorescence studies of endogenous and heterologous APC expression in HCT116 and SW480 cells. Cells were analysed for APC expression via IF using a C-terminal APC-specific primary antibody, and secondary anti-rat polyclonal antibody with a Alexa Fluor 546 conjugate. (A, F and K) HCT116 cells (B-E, G-J and L-O) SW480 cells. SW480 cells were infected with either HVS-GFP-BAC or HVS-GFP-APC at a m.o.i of 0.25. As expected, SW480- and HVS-GFP-BAC-infected cells were negative for APC expression (L and M), whereas infection of SW480 cells with the HVS-GFP-APC clones 4 and 5 resulted in detectable APC expression (N and O). GFP analysis was used to determine viral infection. Scale bar represents $10 \mu \mathrm{m}$. (A-E) DAPI (F-J) Virus expressed GFP (K-L) Alexa Fluor 546.

inserted in an HVS-based vector, and that infection of SW480 cells leads to expression of full length APC protein. To our knowledge this is the first time a complete functional APC gene has been delivered by an infectious viral based vector.

APC expression from HVS-GFP-APC5 reduces $S W 480$ cell proliferation. Release of $\beta$-catenin from its APC-associated inhibition complex leads to increased c-myc expression (10). Therefore, we hypothesised that delivery of the APC cDNA via the HVS-BAC would restore the regulation of c-myc expression and halt SW480 cellular growth. In order to confirm this hypothesis, a cell growth kinetic assay was performed.
Approximately $1 \times 10^{4} \mathrm{SW} 480$ cells were infected with either HVS-GFP-BAC or HVS-GFP-APC5 viruses at a m.o.i of 5. Cell numbers were then quantified at 24-h time intervals. Growth kinetics indicated that the SW480 cells and the HVS-GFP-BAC-infected cells grew at similar rates, whereas SW480 cells that had been infected with the HVS-GFP-APC5 virus grew at a reduced rate. This suggests that restoration of a fully functional APC protein alters the growth kinetics of SW480 cells (Fig. 3).

HVS-GFP-APC5 is stably maintained in SW480 cells. Gene therapy is a treatment administered to the patient with the 


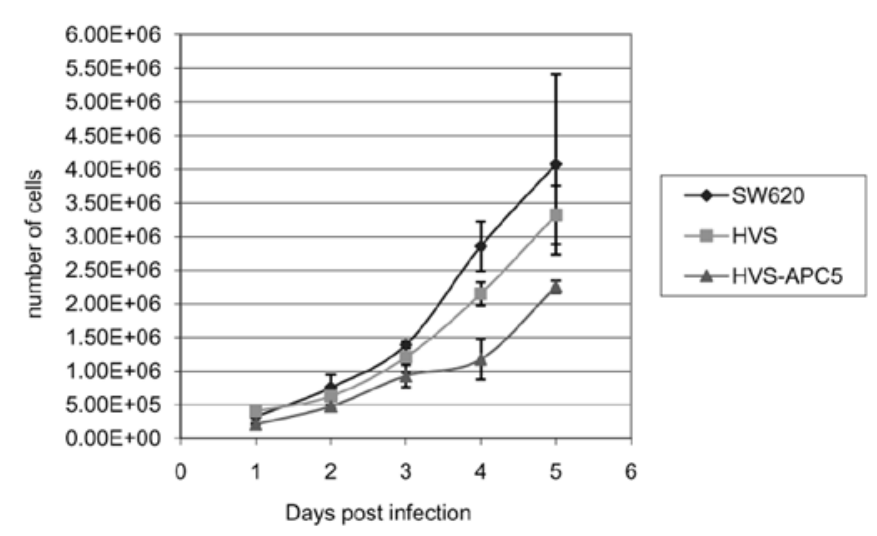

Figure 3. Proliferation of SW480 cells infected with HVS-GFP-BAC or HVS-GFP-APC5 virus. Cells were seeded at a density of $1 \times 10^{4}$ and infected $6 \mathrm{~h}$ post-seeding. Samples were incubated for a further $18 \mathrm{~h}$ before cell numbers were quantified at 24-h time intervals. Error bars represent standard deviation $(n=3)$.

intention of prolonged therapeutic benefit. Therefore it is vital that the gene therapy vector can persist in the target cells and continue to express the therapeutic gene. To assess the potential for long-term vector maintenance and transgene expression, SW480 stable cell lines were generated containing either latent non-integrated HVS-GFP-BAC or HVS-GFP-APC5 episomes. These cell lines would provide information regarding the functional ability of the APC mini-gene to confer sustained reduction of cellular growth.

SW480 cells were infected with either HVS-GFP-BAC or HVS-GFP-APC5 viruses at a m.o.i of $2.48 \mathrm{~h}$ post-infection, GFP fluorescence indicated successful infection with both HVS recombinant viruses. Hygromycin B was then used to select for infected cells. Cells were continually expanded under selection until $100 \%$ of the cells expressed GFP (Fig. 4A), a process that took $\sim 6$ weeks. The stable cell lines containing the HVS-GFP-BAC or the HVS-GFP-APC5 episomes were termed SW480HVS and SW480HVSAPC5, respectively.

The HVS-BAC genome can be maintained in a latent state within SW480 cells over a period of several months (27). However it was not known whether the increased size of the HVS-APC genome affects the genomic stability within cells or whether integration might occur at homologous APC regions. Therefore, an episomal assay was performed on the SW480HVSAPC5 cells to determine whether the enlarged HVS-GFP-APC genome was maintained as a non-integrated episome after 8 weeks of culture post original selection. Rescued BAC DNA was used to transform DH10 $\beta$ electro-competent $E$. coli. DNA isolated from chloramphenicol- and kanamycin-resistant colonies was then screened via restriction digest with I-PpoI and visualised by PFGE (Fig. 4B). Results indicated that upon digestion, BAC episomes were rescued from all samples, containing the 10-kb APC mini-gene DNA fragment. This suggested that the HVS-GFP-BAC-APC5 vector remained as a non-integrated episome within the SW480 cells during the course of the experiment.

HVS-GFP-APC5 maintains long-term transgene expression in SW480 cells. Previous research has shown that therapeutic

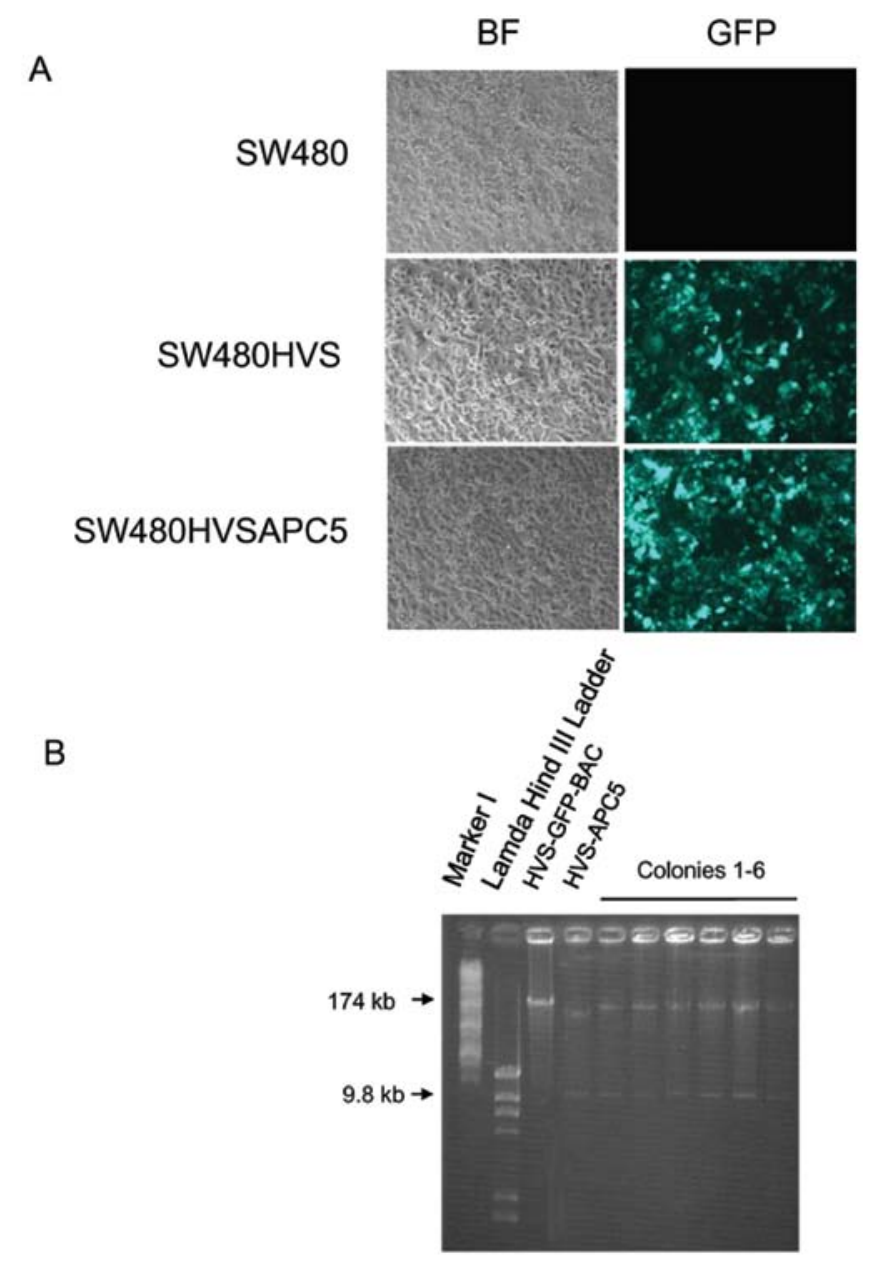

Figure 4. (A) Formation of stable cell lines expressing HVS-GFP-BAC or HVS-GFP-APC5. SW480 cells were infected with HVS-GFP-BAC or HVSGFP-BAC-APC5 viruses and then expanded through routine tissue culture in the presence of hygromycin (B) in order to remove any non-fluorescing cells. GFP fluorescence was observed in these stable cell lines throughout the 8-week time course. (B) Episome rescue studies were performed on SW480HVSAPC cells and analysed via I-ppo-I restriction digest and PFGE. These results show that the vectors were stably maintained in an episomal state for 8 weeks. However this does completely rule out the possibility of some level of genome integration.

cDNA transgene expression can become silenced over long periods in vitro $(33,34)$. Therefore, we used the SW480HVS and SW480HVSAPC stable cell lines to analyse any longterm effect of expressing the APC cDNA on cellular growth. SW480, SW480HVS or SW480HVSAPC5 cells $\left(1 \times 10^{4}\right)$ that had been stably grown for 2 months were seeded in parallel. Cell numbers were then quantified at 24-h time intervals (Fig. 5A). SW480 and SW480HVS cells grew at a similar rate. In contrast the SW480HVSAPC5 cells exhibited significantly slower growth. These results show a similar trend to that observed when naïve SW480 cells were infected with APC-expressing virus and immediately quantified (Fig. 3), suggesting that long-term expression of APC cDNA from an HVS-based vector could have a positive therapeutic benefit.

A common treatment for sporadic colorectal polyps or individual polyps associated with FAP is endoscopic or surgical removal from the affected region of the colon. In order to assess the effects of expression of the APC mini- 


\section{A}

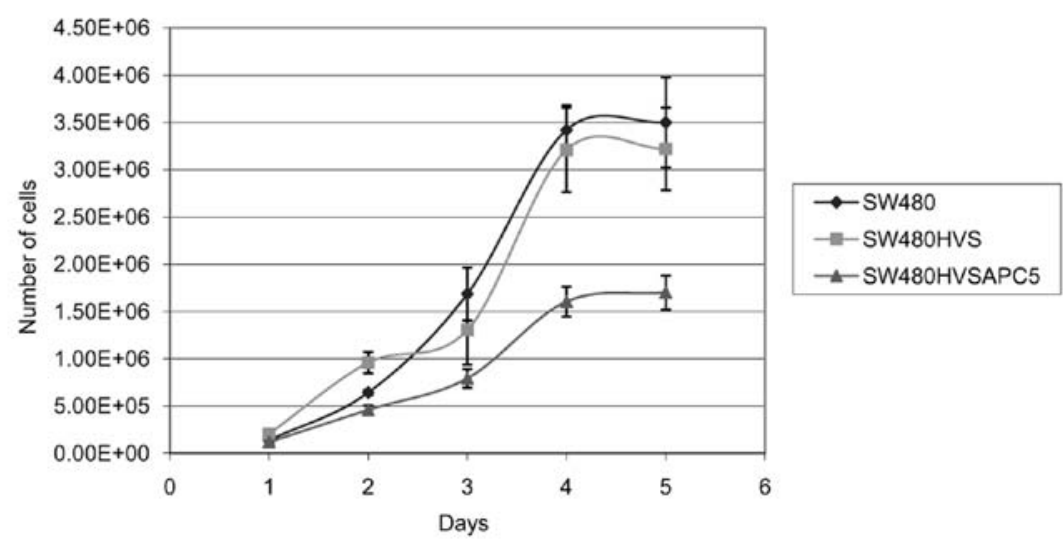

B

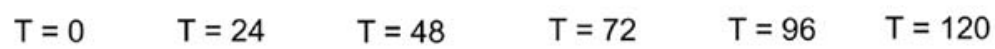

\section{SW480}
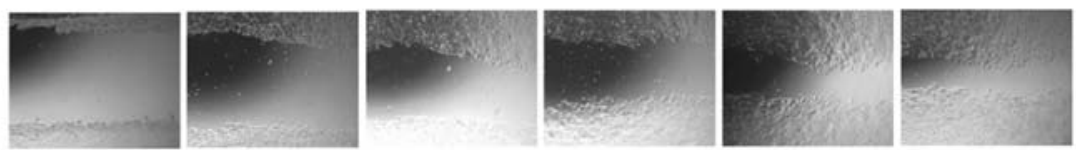

SW480HVS
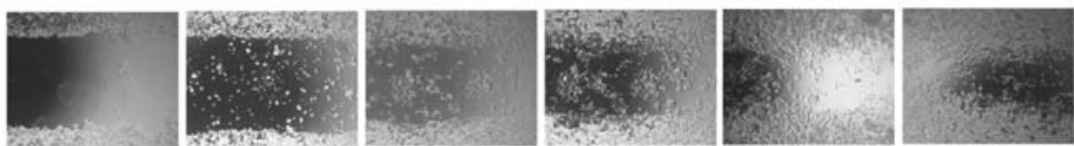

SW480HVSAPC5
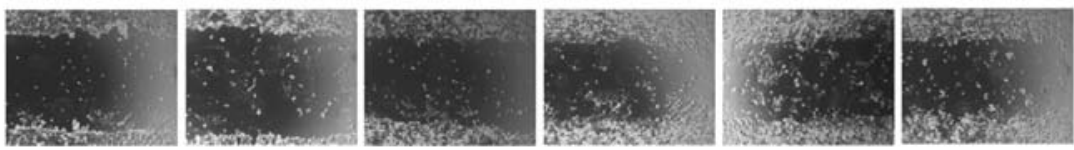

Figure 5. Stably infected SW480 cells show reduced proliferation. (A) SW480, SW480HVS or SW480HVSAPC5 cells (1x10 ${ }^{4}$ ) were seeded and quantified at 24-h time intervals, for a period of 5 days. The SW480 cells infected with the APC-expressing vector show a marked decrease in cell number compared to controls. Error bars represent standard deviation. (B) Monolayer wounding of SW480 cell lines. Confluent SW480, SW480HVS or SW480HVSAPC5 stable cell lines $(100 \%)$ were wounded with a 1-ml pipette tip. Over 5 days the SW480 and SW480HVS cells began to migrate back into the cleared area. However SW480HVSAPC5 cell migration was substantially reduced. Samples were photographed at 24-h intervals. T, hours post-wounding. Images were recorded using bright-field microscopy.

gene on wounded cells, a second growth assay was employed, assessing the ability of cells to migrate and proliferate into a mono-layer wound, thereby determining the likelihood of polyp growth after surgical intervention and HVS gene therapy. SW480, SW480HVS, SW480HVSAPC5 cells were seeded into $35-\mathrm{mm}$ tissue culture dishes and allowed to reach $100 \%$ confluence. Cells were then wounded by scratching a 1-ml pipette tip through the cell sheet. Post-wounding samples were photographed at time point 0 and at 24-h intervals for a period of 5 days. Consistent with the cell growth assay using stable cell lines, SW480 and SW480HVS cell lines migrated and proliferated into the wound at similar rates, whereas SW480HVSAPC5 migrated at a much slower rate (Fig. 5B). This evidence further suggests that restoration of APC expression in SW480 cells may have a therapeutic effect.
HVS-GFP-APC5 virus causes a reduction in SW620 cell proliferation. We next assessed whether HVS-APC could inhibit growth of metastatic colorectal tumour cells. SW620 cells are a metastatic cell line derived from the same patient from whom the SW480 cells were isolated. The cells are APC-deficient but have other established genetic changes which reflect their metastatic nature and more aggressive phenotype (38). Therefore, these cells provide an ideal model in which further study HVS-based APC-mediated gene therapy.

A cellular growth assay was performed using $1 \times 10^{4}$ SW620 cells infected with either HVS-GFP-BAC virus or HVSGFP-APC5 virus at a m.o.i of 5. Cell numbers were quantified at 24-h time intervals. Cell growth of uninfected SW620 cells and HVS-GFP-BAC infected cells was comparable, although there was a slight decrease in the amount of cell proliferation in 


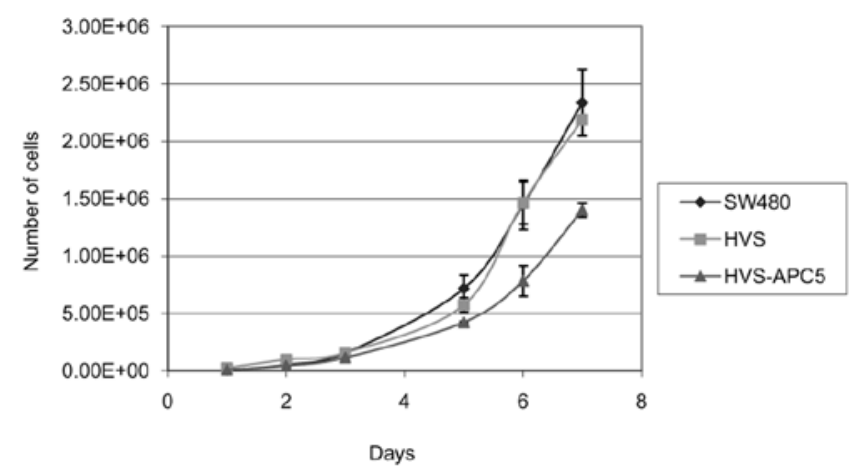

Figure 6. Proliferation of SW620 cells infected with HVS-GFP-BAC or HVSGFP-APC5 viruses. Cells were seeded at a density of $1 \times 10^{4}$ and infected $6 \mathrm{~h}$ post-seeding. Samples were incubated for a further $18 \mathrm{~h}$ before cell numbers were quantified at 24-h time intervals. Error bars represent standard deviation $(n=3)$.

the HVS-GFP-BAC infected cells. However, cells that had been infected with HVS-GFP-APC5 virus proliferated more slowly than the control cell lines (Fig. 6). The data suggests that delivery of the APC mini-gene to metastatic cells with APC mutations may reduce their growth proliferation characteristics.

\section{Discussion}

HVS has the potential to be used as a novel gene therapy vector $(21,33,37)$. Its episomal maintenance properties combined with its natural tropism for human colorectal cells make it an ideal candidate vector for treating colorectal cancer (38). In order to assess this potential, we cloned the APC mini-gene into the HVS-GFP-BAC genome and tested the effects of this HVS-GFP-APC virus by measuring the growth kinetics of SW480 cells. In spite of the addition of $10 \mathrm{~kb}$ to the viral genome, HVS-GFP-APC DNA was efficiently packaged into virions which were able to infect $\mathrm{CRC}$ cell lines. Moreover, expression of the full length APC protein from the HVS-BAC seemed to provide a therapeutic effect in these cells. Furthermore, we also demonstrated that stably infected SW480HVSAPC5 cells have a reduced ability to proliferate and migrate, for over a period of 2 months, by way of a cell monolayer wounding assay similar to that described by Faux et al (19).

Most current viral vectors have insufficient capacity for native genomic gene sequences. Vectors expressing the cDNAs of therapeutic genes therefore provide a method for overcoming the bottle-neck in vector packaging capacity. However, studies using the 7-kb cDNA of the blood clotting factor, factor VIII, show that expression of cDNA may lead to gene silencing over long periods as splicing is an integral part of cellular mRNA processing $(33,34)$. Although APC cDNA was used in this study, HVS has a coding genome of $\sim 112 \mathrm{~kb}$ and therefore the virion has the packaging potential to deliver the full $98-\mathrm{kb}$ APC genomic locus. Increasing the genome size will reduce the number of terminal repeats (TR), which are typically 50 repeats of $1.4 \mathrm{~kb}$ (39), therefore allowing excess coding DNA to be packaged. Although a number of TRs are required for episomal persistence, the minimum number needed has been determined to be only 4 repeats $(40,41)$. As HVS can have varied TR number profiles, there may be potential for increasing the transgene capacity of the genome by $44.7 \mathrm{~kb}$ beyond the normal 112-kb size, as TR regions may not get amplified to such an extent in the presence of a larger genome $(31,42)$.

Packaging in excess of the WT-genome will ultimately have a finite upper limit. To overcome the limitations imposed by the virion capsid, another method to increase transgene packaging capacity would be the removal of viral gene sequences and generation of amplicon virus-like particles (ampVLPs). Construction of a first generation HVSampVLP has recently been achieved (37). Amplicons contain the minimal components for effective gene therapy, namely the therapeutic expression cassette, plus the viral elements required for episomal maintenance and packaging. Structural viral proteins are provided in trans by an attenuated helper virus (43). Amplicon systems were originally developed using Herpes simplex virus (HSV)-1. However, other herpesviruses have also been used (44-47). Although the HVS amplicon retained some viral gene sequences, over $50 \mathrm{~kb}$ of the $\sim 112 \mathrm{~kb}$ genome was removed, spanning open-reading frames 19-62 $(37,48)$. This system provides a much greater transgene capacity than the HVS-GFP-BAC, and with further refinement, has a theoretical capacity of $\sim 143 \mathrm{~kb}$ which would be sufficient for delivery of the genomic APC locus.

Although no apparent silencing effects were observed with the HVS-GFP-APC vectors over 8 weeks, more research needs to be carried out to assess transgene expression over longer periods. It is hypothesised that the use of full genomic loci would alleviate any potential problems associated with any fall off in cDNA expression. However, cDNA transgenes are invaluable tools providing proof of principle in the short-term and they appear to provide acute therapeutic benefit.

Our vector appears to express a functional APC protein, but does not completely inhibit SW480 cellular growth. This may be due to the promoter used to express the APC cDNA. The design of the APC cassette utilising the TCF promoter was intended to achieve a self-regulating, natural feedback mechanism, providing appropriate levels of APC-mediated $\beta$-catenin degradation. However, this system may be regulated too tightly, and APC levels may be continuously in flux. Therefore protein levels may not reach the threshold needed to fully counter-act the oncogenic potential of a continuously active Wnt signalling pathway. To determine whether this is the case, further work is now being performed to replace the TCF promoter with a more traditional, continually active promoter such as CMV. Alternatively there may be other pathways involved in SW480 oncogenesis and restoration of the APC element in the Wnt signalling pathway may not be enough alone to inhibit replication or induce apoptosis.

In conclusion, we have developed what we believe to be the first infectious viral-based vector expressing APC and have demonstrated that infection with this vector reduces the proliferation and migration of CRC cells in vitro. This HVS-GFP-APC virus, with further development, could be a powerful agent against $\mathrm{CRC}$ and provides a proof of principle for gene therapy using this HVS vector platform. HVS vectors, although still relatively undeveloped compared to HSV-1 and adenoviral vectors, possess a distinct set of characteristics including large transgene capacity, persistence in dividing cell populations and broad cell tropism, which warrants their use in further gene therapy studies. 


\section{Acknowledgements}

This work was supported by the University of Leeds Interdisiplinary Institute in Bionanoscience and Cancer Research $\mathrm{UK}$. AW is a recipient of a BBSRC research development fellowship. We would like to thank Dr Ewan Morrison (Leeds Institute of Molecular Medicine) for the generous gift of the APC antibodies.

\section{References}

1. Cunningham D, Atkin W, Lenz $\mathrm{H}$, et al: Colorectal cancer Lancet 375: 1030-1047, 2010.

2. de la Chapelle A: Genetic predisposition to colorectal cancer. Nat Rev Cancer 4: 769-780, 2004.

3. Half E, Bercovich D and Rozen P: Familial adenomatous polyposis. Orphanet J Rare Dis 4: 22, 2009.

4. Bodmer WF, Bailey CJ, Bodmer J, et al: Localization of the gene for familial adenomatous polyposis on chromosome 5. Nature 328: 614-616, 1987.

5. Kinzler KW, Nilbert MC, Su LK, et al: Identification of FAP locus genes from chromosome 5q21. Science 253: 661-665, 1991

6 . Fodde R: The multiple functions of tumour suppressors: it's all in APC. Nat Cell Biol 5: 190-192, 2003.

7. Joslyn G, Richardson DS, White R and Alber T: Dimer formation by an N-terminal coiled coil in the APC protein. Proc Natl Acad Sci USA 90: 11109-11113, 1993.

8. Taipale $J$ and Beachy PA: The Hedgehog and Wnt signalling pathways in cancer. Nature 411: 349-354, 2001.

9. Hinoi T, Yamamoto H, Kishida M, Takada S, Kishida S and Kikuchi A: Complex formation of adenomatous polyposis coli gene product and axin facilitates glycogen synthase kinase-3 beta-dependent phosphorylation of beta-catenin and downregulates beta-catenin. J Biol Chem 275: 34399-34406, 2000.

10. He TC, Sparks AB, Rago C, et al: Identification of c-MYC as a target of the APC pathway. Science 281: 1509-1512, 1998.

11. Tetsu $\mathrm{O}$ and McCormick F: Beta-catenin regulates expression of cyclin D1 in colon carcinoma cells. Nature 398: 422-426, 1999.

12. Mann B, Gelos M, Siedow A, et al: Target genes of beta-catenin$\mathrm{T}$ cell-factor/lymphoid-enhancer-factor signaling in human colorectal carcinomas. Proc Natl Acad Sci USA 96: 1603-1608, 1999.

13. Miyoshi Y, Nagase H, Ando H, et al: Somatic mutations of the APC gene in colorectal tumors: mutation cluster region in the APC gene. Hum Mol Genet 1: 229-233, 1992.

14. Lagarde A, Rouleau E, Ferrari A, et al: Germline APC mutation spectrum derived from 863 genomic variations identified through a 15-year medical genetics service to French patients with FAP. J Med Genet 10: 721-722, 2010.

15. Fearon ER and Vogelstein B: A genetic model for colorectal tumorigenesis. Cell 61: 759-767, 1990.

16. Westbrook CA, Chmura SJ, Arenas RB, Kim SY and Otto G: Human APC gene expression in rodent colonic epithelium in vivo using liposomal gene delivery. Hum Mol Genet 3: 2005-2010, 1994 .

17. Hargest R, Eldin A and Williamson R: Gene therapy for familial adenomatous polyposis. Prolonged expression of the adenomatous polyposis coli gene after lipofection into mouse colon in vivo. Adv Exp Med Biol 451: 385-391, 1998.

18. Lee J, Hargest R, Wasan H and Phillips RK: Liposome-mediated adenomatous polyposis coli gene therapy: a novel anti-adenoma strategy in multiple intestinal neoplasia mouse model. Dis Colon Rectum 47: 2105-2113, 2004.

19. Faux MC, Ross JL, Meeker C, et al: Restoration of full-length adenomatous polyposis coli (APC) protein in a colon cancer cell line enhances cell adhesion. J Cell Sci 117: 427-439, 2004.

20. Calderwood M, White RE, Griffiths RA and Whitehouse A: Open reading frame 73 is required for herpesvirus saimiri A11-S4 episomal persistence. J Gen Virol 86: 2703-2708, 2005.

21. Griffiths RA, Boyne JR and Whitehouse A: Herpesvirus saimiribased gene delivery vectors. Curr Gene Ther 6: 1-15, 2006.

22. Stevenson AJ, Cooper M, Griffiths JC, et al: Assessment of Herpesvirus saimiri as a potential human gene therapy vector. J Med Virol 57: 269-277, 1999.

23. Hall KT, Giles MS, Goodwin DJ, et al: Analysis of gene expression in a human cell line stably transduced with herpesvirus saimiri. J Virol 74: 7331-7337, 2000.
24. Stevenson AJ, Frolova-Jones E, Hall KT, et al: A herpesvirus saimiri-based gene therapy vector with potential for use in cancer immunotherapy. Cancer Gene Ther 7: 1077-1085, 2000.

25. Stevenson AJ, Giles MS, Hall KT, et al: Specific oncolytic activity of herpesvirus saimiri in pancreatic cancer cells. Br J Cancer 83: 329-332, 2000.

26. Smith PG, Oakley F, Fernandez M, Mann DA, Lemoine NR and Whitehouse A: Herpesvirus saimiri-based vector biodistribution using noninvasive optical imaging. Gene Ther 12: 1465-1476, 2005.

27. Smith PG, Coletta PL, Markham AF and Whitehouse A: In vivo episomal maintenance of a herpesvirus saimiri-based gene delivery vector. Gene Ther 8: 1762-1769, 2001.

28. Smith PG, Burchill SA, Brooke D, Coletta PL and Whitehouse A: Efficient infection and persistence of a herpesvirus saimiri-based gene delivery vector into human tumor xenografts and multicellular spheroid cultures. Cancer Gene Ther 12: 248-256, 2005.

29. Hargest R and Williamson R: Expression of the APC gene after transfection into a colonic cancer cell line. Gut 37: 826-829, 1995.

30. Goss KH and Groden J: Biology of the adenomatous polyposis coli tumor suppressor. J Clin Oncol 18: 1967-1979, 2000.

31. White RE, Calderwood MA and Whitehouse A: Generation and precise modification of a herpesvirus saimiri bacterial artificial chromosome demonstrates that the terminal repeats are required for both virus production and episomal persistence. J Gen Virol 84: 3393-3403, 2003.

32. Turrell S and Whitehouse A: Mutation of herpesvirus saimiri ORF51 glycoprotein specifically targets infectivity to hepatocellular carcinoma cell lines. J Biomed Biotechnol (In press).

33. Lynch CM, Israel DI, Kaufman RJ and Miller AD: Sequences in the coding region of clotting factor VIII act as dominant inhibitors of RNA accumulation and protein production. Human Gene Therapy 4: 259-272, 1993.

34. Hoeben R, Fallaux F, Cramer S, et al: Expression of the bloodclotting factor-VIII cDNA is repressed by a transcriptional silencer located in its coding region. Blood 85: 2447-2454, 1995.

35. Leibovitz A, Stinson JC, McCombs WB 3rd, McCoy CE, Mazur KC and Mabry ND: Classification of human colorectal adenocarcinoma cell lines. Cancer Res 36: 4562-4569, 1976.

36. Hong Y, Macnab S, Lambert L, Tuner A, Whitehouse A and Usmani B: Herpesvirus saimiri-based endothelin-converting enzyme-1 shRNA expression decreases prostate cancer cell invasion and migration. Int J Cancer (In press)

37. Macnab S, White R, Hiscox J and Whitehouse A: Production of an infectious Herpesvirus saimiri-based episomally maintained amplicon system. J Biotechnol 134: 287-296, 2008.

38. Whitehouse A: Herpesvirus saimiri: a potential gene delivery vector (review). Int J Mol Med 11: 139-148, 2003.

39. Stamminger T, Honess RW, Young DF, Bodemer W, Blair ED and Fleckenstein B: Organization of terminal reiterations in the virion DNA of herpesvirus saimiri. J Gen Virol 68: 1049-1066, 1987.

40. Griffiths R and Whitehouse A: Herpesvirus saimiri episomal persistence is maintained via interaction between open reading frame 73 and the cellular chromosome-associated protein MeCP2. J Virol 81: 4021-4032, 2007.

41. Calderwood MA, Hall KT, Matthews DA and Whitehouse A: The herpesvirus saimiri ORF73 gene product interacts with host-cell mitotic chromosomes and self-associates via its $\mathrm{C}$ terminus. J Gen Virol 85: 147-153, 2004

42. White RE, Carline L and Allday MJ: Mutagenesis of the herpesvirus saimiri terminal repeat region reveals important elements for virus production. J Virol 81: 6765-6770, 2007.

43. Epstein AL: HSV-1-based amplicon vectors: design and applications. Gene Ther 12 (Suppl 1): S154-S158, 2005.

44. Spaete RR and Frenkel N: The herpes simplex virus amplicon: a new eucaryotic defective-virus cloning-amplifying vector. Cell 30: 295-304, 1982

45. Saeki Y, Fraefel C, Ichikawa T, Breakefield XO and Chiocca EA: Improved helper virus-free packaging system for HSV amplicon vectors using an ICP27-deleted, oversized HSV-1 DNA in a bacterial artificial chromosome. Mol Ther 3: 591-601, 2001.

46. White RE, Wade-Martins R and James MR: Infectious delivery of 120-kilobase genomic DNA by an Epstein-Barr virus amplicon vector. Mol Ther 5: 427-435, 2002.

47. Borst EM and Messerle M: Construction of a cytomegalovirusbased amplicon: a vector with a unique transfer capacity. Hum Gene Ther 14: 959-970, 2003

48. Macnab S and Whitehouse A: Progress and prospects: human artificial chromosomes. Gene Therapy 16: 1180-1188, 2009. 